\title{
Prevention of lodinated Contrast Media-Induced Hyperthyroidism in Patients with Euthyroid Goiter
}

\author{
Katarzyna Pelewicz $^{\mathrm{a}}$ Rafał Wolny ${ }^{\mathrm{b}}$ Tomasz Bednarczuk $^{\mathrm{a}}$ Piotr Miśkiewicz $^{\mathrm{a}}$ \\ aDepartment of Internal Medicine and Endocrinology, Medical University of Warsaw, Warsaw, Poland; \\ ${ }^{b}$ Department of Interventional Cardiology and Angiology, National Institute of Cardiology, Warsaw, Poland
}

\section{Keywords}

lodinated contrast media $\cdot$ lodine-induced hyperthyroidism . Nodular goiter $\cdot$ Sodium perchlorate $\cdot$ Thiamazole

\begin{abstract}
Introduction: lodinated contrast media (ICM)-induced hyperthyroidism is an underestimated, potentially severe condition; however, its prevention has not been sufficiently investigated. The aim of this study was to evaluate the influence of ICM on thyroid status, the advantages of prophylactic therapy for iodine-induced hyperthyroidism $(\mathrm{IIH})$ in patients with euthyroid goiter and cardiovascular comorbidities, and the association between the incidence of $\mathrm{IIH}$ and thyroid volume. Methods: Thirty-six euthyroid patients undergoing procedures involving ICM administration were divided into 2 groups: the first group $(n=13)$ received prophylactic treatment with thiamazole or thiamazole combined with sodium perchlorate during ICM exposure; the second group $(n=23)$ did not receive prophylaxis. Thyroid-stimulating hormone levels were evaluated before and after ICM, and thyroid hormone levels were assessed after ICM at different points in time. The morphology of the thyroid was evaluated by ultrasonography. Results: Twenty-one patients (58\%) developed hyperthyroidism after ICM. Hyperthyroidism was observed more frequently in the group without prophylactic treatment
\end{abstract}

than in the group with prophylaxis (65 vs. $15 \%$, respectively; $p=0.006$ ). No cases of overt hyperthyroidism were observed in the group receiving thiamazole with sodium perchlorate. IIH persisted for a median time of 52.5 days. Larger thyroid volume was associated with a significantly higher occurrence of ICM-induced hyperthyroidism ( $p=0.04)$. Conclusions: $\mathrm{Pa}$ tients with euthyroid goiter receiving ICM are at risk of developing hyperthyroidism. The occurrence of hyperthyroidism after ICM in euthyroid patients with goiter is higher in those with larger thyroid volume. The frequency of ICM-induced hyperthyroidism in euthyroid patients with goiter is lower in those receiving prophylactic therapy with thiamazole in monotherapy or in combination with sodium perchlorate than in those not receiving prophylactic treatment.

\footnotetext{
(c) 2021 European Thyroid Association Published by S. Karger AG, Basel
}

\section{Introduction}

The proper synthesis of thyroid hormones is dependent on adequate iodine supply. The mean daily iodine intake recommended by the World Health Organization

Clinical trial: lodinated Contrast Media Induced Hyperthyroidism, NCT04304794, https://clinicaltrials.gov/ct2/show/NCT04304794? cond $=$ NCT04304794\&draw $=2 \&$ rank $=1$.
Correspondence to:

Piotr Miśkiewicz, piotr.miskiewicz@ wum.edu.pl 
is $150 \mu \mathrm{g}$ [1]. Iodinated contrast media (ICM) typically contain $13,500 \mu \mathrm{g}$ of free iodine and 15-60 g of bound iodine [2], an amount well above the acceptable upper level. In the case of excess iodine, thyroid discontinues the release of hormones (the acute Wolff-Chaikoff effect). This effect is usually transient, although it can persist causing hypothyroidism. A pathologic response to the exogenous iodine load may lead to iodine-induced hyperthyroidism (IIH), known as the Jod-Basedow phenomenon. IIH is infrequent, but elderly patients and individuals with autonomously functioning nodular goiters are at higher risk of developing this dysfunction [3]. The risk of goiter development is higher in the area of iodine deficiency [4]. Deficient iodine intake may impact the secretion of thyroid-stimulating hormone (TSH), causing a rise of the iodine uptake, thyroid hypertrophy, and hyperplasia $[5,6]$. In mild to moderate iodine deficiency, the thyroid is able to maintain euthyroidism in most individuals due to increased activity; however, as a consequence, it may lead to the development of multinodular goiter [7]. After the cessation of salt iodization in Poland in 1980, mild to moderate iodine deficiency was detected. The prevalence of goiter in adults was assessed as 30\% [8]. Consequently, the voluntary model of iodine prophylaxis conducted until then was judged unsuccessful, and Poland introduced obligatory iodization of household salt in 1997. In 2002, Poland was declared as iodine sufficient, and in the recent studies, an adequate iodine intake in the general population was presented $[9,10]$. However, due to iodine deficiency persisting for more than a decade, the rate of elderly with undiagnosed goiter may be significant as the prevalence of nodules in adults older than 50 years is not reduced with iodine repletion [4].

According to recent studies, the risk of ICM-induced hyperthyroidism appears to be low in the general population [11]. Currently, there are no specific guidelines concerning IIH prophylactic therapy. Due to scarce evidence on the advantages of prophylactic treatment, we performed a retrospective study of the effect of the prophylactic application of antithyroid drugs (ATDs) during excessive iodine exposure in patients with euthyroid goiter.

\section{Materials and Methods}

\section{Patients}

Our retrospective study included patients with ICM exposure admitted to the National Institute of Cardiology between January 2015 and November 2019. Inclusion criteria were euthyroidism at baseline (defined as TSH level within the reference range); diffuse or multinodular goiter; ICM exposure; and time of observation after ICM exposure longer than 4 weeks. Exclusion criteria were: Graves' disease; administration of thyroid hormones, glucocorticoids, iodine-containing medications (e.g., amiodarone), or iodine contrast agent within the past 6 months.

Finally, 36 patients were included in the study. All patients presented multiple comorbidities, mainly cardiovascular, such as congestive heart failure, ischemic heart disease, and valvulopathies. A number of individuals (25\%) were treated with a low dose of thiamazole (2.5-5.0 $\mathrm{mg}$ /day) within 3 months prior to admission. Institutional Ethics Committee approval was obtained for retrospective data collection.

\section{Study Design}

Patients were divided into 2 subsets on the basis of received therapy:

- the group with prophylaxis $(n=13)$ received thiamazole with $(n=6)$ or without $(n=7)$ sodium perchlorate, 1 day prior to ICM and for 14 days after for thiamazole (20-40 mg/daily) and at least 10 days after for sodium perchlorate $(300 \mathrm{mg} 3$ times a day) and

- the group with prophylaxis $(n=13)$ received thiamazole with $(n=6)$ or without $(n=7)$ sodium perchlorate, 1 day prior to ICM and for 14 days after for thiamazole (20-40 mg/daily) and at least 10 days after for sodium perchlorate $(300 \mathrm{mg} 3$ times a day) andthe group without prophylaxis $(n=23)$ received no prophylactic treatment.

Patients diagnosed with goiter who were prepared for procedures involving ICM administration were consulted by an endocrinologist and given prophylactic treatment. Patients included in the study who did not receive prophylactic therapy before ICM injection were not assessed prior by an endocrinologist, due to urgent indications for scans or procedures involving ICM application. Seven individuals received only thiamazole as prophylaxis due to a lack of sodium perchlorate in the institute at the time. Each individual had TSH and Cr level measured at baseline. There was no significant difference between the groups with regard to age, sex, TSH level at baseline, and goiter size. The levels of TSH, free triiodothyronine (fT3), and free thyroxine (fT4) were evaluated at different points in time after ICM.

\section{Laboratory Tests}

Normal thyroid function was defined as TSH levels between 0.27 and $4.20 \mu \mathrm{IU} / \mathrm{mL}$. The reference range for fT4 and fT3 was 0.93-1.7 ng/dL and 2.0-4.4 pg/mL, respectively. The measuring range and limit of detection (as given by providers) for TSH were $0.005-100 \mu \mathrm{IU} / \mathrm{mL}$ and $0.005 \mu \mathrm{IU} / \mathrm{mL}$, respectively, for fT4 $0.5-$ $100 \mathrm{pmol} / \mathrm{L}$ and $0.5 \mathrm{pmol} / \mathrm{L}$, respectively, and for fT3 $0.4-50$ $\mathrm{pmol} / \mathrm{L}$ and $0.6 \mathrm{pmol} / \mathrm{L}$, respectively. The reference range for $\mathrm{Cr}$ was $0.5-0.9 \mathrm{mg} / \mathrm{dL}$ (women) and $0.7-1.2 \mathrm{mg} / \mathrm{dL}$ (men). TSH, fT4, and $\mathrm{fT} 3$ levels were measured using an electrochemiluminescence immunoassay on Cobas 6,000 analyzer (Roche Diagnostics, Mannheim, Germany). The Cr level was measured using the Jaffe colorimetric method on Cobas 6,000 analyzer (Roche Diagnostics, Mannheim, Germany). Subclinical hyperthyroidism was diagnosed on the basis of suppressed TSH as well as fT4 and fT3 values within the reference range. Overt hyperthyroidism was defined as a TSH level below the lower limit and elevated fT4 and/or fT3 concentrations. Subclinical hypothyroidism was identified as an elevated TSH level as well as fT4 and fT3 levels within the reference range.
Prevention of ICM-Induced

Hyperthyroidism in Euthyroid Goiter
Eur Thyroid J 2021;10:306-313

DOI: $10.1159 / 000515126$ 
Table 1. Baseline characteristics of patients

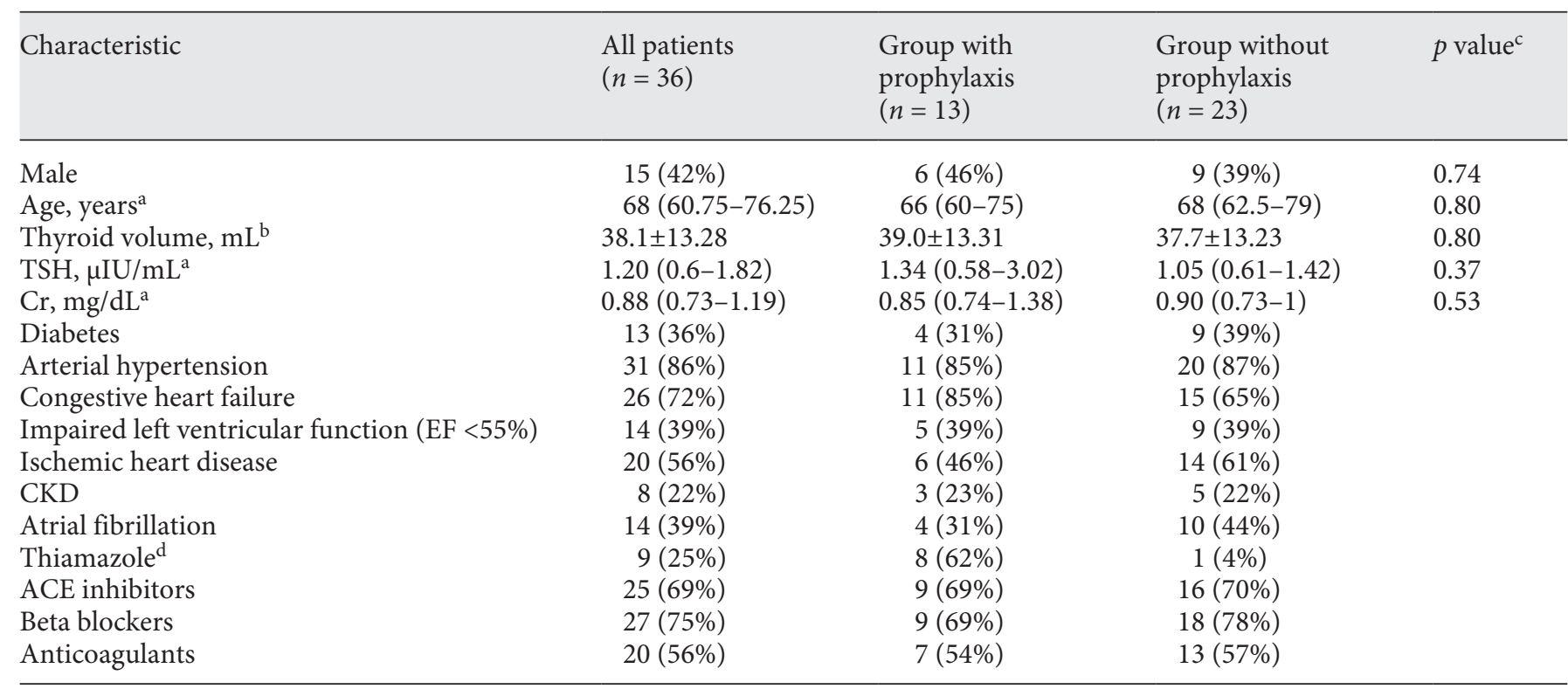

CKD, chronic kidney disease; EF, ejection fraction; ICM, iodinated contrast medium; TSH, thyroid-stimulating hormone (reference range: $0.27-4.2 \mu \mathrm{IU} / \mathrm{mL})$. Group with prophylaxis, patients who received thiamazole with $(n=6)$ or without $(n=7)$ sodium perchlorate: 1 day prior to ICM and for 14 days after for thiamazole (20-40 mg/day) and at least 10 days after for sodium perchlorate (900 mg/day). Group without prophylaxis, patients who received no prophylactic treatment. Cr reference range: $0.5-0.9 \mathrm{mg} / \mathrm{dL}$ (women) and 0.7-1.2 $\mathrm{mg} / \mathrm{dL}$ (men). ${ }^{\mathrm{a}}$ Data are presented as medians (interquartile range). ${ }^{\mathrm{b}}$ Data are presented as means ( \pm standard deviation). ${ }^{\mathrm{c}} p$ values for group with prophylaxis versus group without prophylaxis. ${ }^{\mathrm{d}}$ Number of individuals treated with low dose of thiamazole (2.5-5.0 mg/ day) within 3 months prior to admission.

\section{Ultrasound of the Thyroid}

Thyroid morphology was assessed in each patient using ultrasound during hospitalization at the National Institute of Cardiology on GE Logiq 9 ultrasound system (GE Vingmed Ultrasound, Horten, Norway). The volume of each thyroid lobe was estimated using the formula: height $\times$ width $\times$ depth $\times$ a correction factor (0.5) [12]. The thyroid volume was the sum of the left and right lobe volumes. Enlargement of the gland was defined as a volume exceeding $18 \mathrm{~mL}$ in women and $25 \mathrm{~mL}$ in men [13]. Diffuse goiter was diagnosed if ultrasonography detected thyroid enlargement without the presence of nodules. Multinodular goiter was diagnosed if the nodules were reported.

\section{ICM Exposure}

Patients were exposed to ICM at the National Institute of Cardiology during diagnostic or treatment procedures due to cardiovascular diseases. ICM was administered during computed tomography, coronary angiography/angioplasty, or transcatheter aortic valve implantation. Iomeron 350 (iomeprol) or Ultravist 370 (iopromide) ICM with the amount of 350 and $370 \mathrm{mg}$ iodine per $\mathrm{ml}$, respectively, were used in the procedures.

\section{Statistical Analysis}

Statistical analysis was performed using the GraphPad Prism 8.0 software. Results were expressed as medians (interquartile range), with the exception of the thyroid volume, which was expressed as a mean ( \pm standard deviation). Categorical variables were expressed as numbers $(n)$ and percentages (\%). The Wilcoxon test was used for comparing the TSH levels before and after the ICM. Unpaired Student's $t$ test, Mann-Whitney U-test, and Fisher's exact test were used to assess differences between groups. All results were considered statistically significant at $p<0.05$.

\section{Results}

The baseline characteristics of patients are presented in Table 1 . We observed 21/36 (58\%) cases of hyperthyroidism following ICM, 17 (47\%) cases of overt hyperthyroidism, and $4(11 \%)$ cases of subclinical hyperthyroidism. Subclinical hypothyroidism was registered in 2 individuals. Overt hyperthyroidism was more frequent in the group without prophylaxis (15/23 patients, 65\%) than in the group with prophylaxis $(2 / 13$ patients, $15 \%)(p=0.006$; Table 2). None of the patients who had received thiamazole in combination with sodium perchlorate as prophylaxis developed overt hyperthyroidism, while 1 individual developed subclinical hyperthyroidism. All patients diagnosed with overt hyperthyroidism and 3 patients with subclinical hyperthyroidism were treated with thiamazole in 
Table 2. Thyroid status after ICM

\begin{tabular}{lcllc}
\hline & $\begin{array}{l}\text { All patients } \\
(n=36)\end{array}$ & $\begin{array}{l}\text { Group with } \\
\text { prophylaxis } \\
(n=13)\end{array}$ & $\begin{array}{l}\text { Group without } \\
\text { prophylaxis } \\
(n=23)\end{array}$ & $p$ value $^{\mathrm{a}}$ \\
\hline Euthyroidism & $13(36 \%)$ & $8(62 \%)$ & $5(22 \%)$ & 0.03 \\
Hyperthyroidism & $21(58 \%)$ & $3(23 \%)$ & $18(78 \%)$ & 0.002 \\
Subclinical & $4(11 \%)$ & $1(8 \%)$ & $3(13 \%)$ & 1.00 \\
Overt & $17(47 \%)$ & $2(15 \%)$ & $15(65 \%)$ & 0.006 \\
Subclinical hypothyroidism & $2(6 \%)$ & $2(15 \%)$ & $0(0 \%)$ & 0.12 \\
\hline
\end{tabular}

ICM, iodinated contrast medium. Group with prophylaxis, patients who received thiamazole with $(n=6)$ or without $(n=7)$ sodium perchlorate: 1 day prior to ICM and for 14 days after for thiamazole (20-40 mg/daily) and at least 10 days after for sodium perchlorate $(900 \mathrm{mg} /$ daily). Group without prophylaxis, patients who received no prophylactic treatment. ${ }^{a} p$ value for comparison between group with prophylaxis and group without prophylaxis.

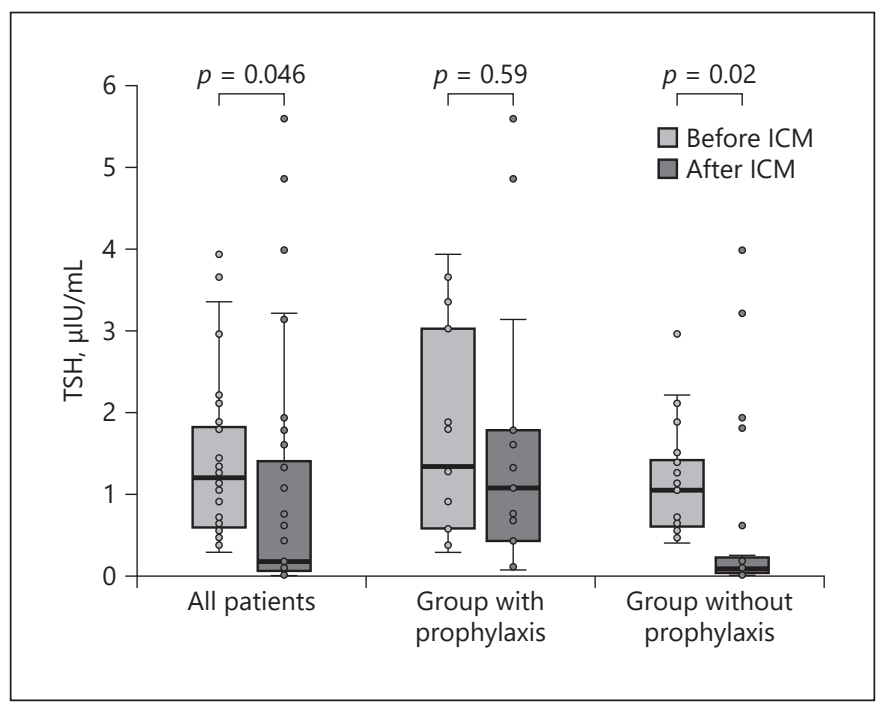

Fig. 1. TSH levels before and after ICM injection. Data are shown as median values (line across the box) with interquartile (25th75th percentile) range (the box). Bullets represent individual TSH levels. Group with prophylaxis, patients who received thiamazole with $(n=6)$ or without $(n=7)$ sodium perchlorate: 1 day prior to ICM and for 14 days after for thiamazole (20-40 mg/daily) and at least 10 days after for sodium perchlorate $(900 \mathrm{mg} /$ daily $)$. Group without prophylaxis, patients who received no prophylactic treatment. ICM, iodinated contrast medium; TSH, thyroid-stimulating hormone (normal range: $0.27-4.2 \mu \mathrm{IU} / \mathrm{mL}$ ).

doses of 20-40 mg per day. The patient from the group with combination prophylactic therapy with thiamazole and sodium perchlorate, who developed subclinical hyperthyroidism, was treated with thiamazole after the cessation of prophylaxis. No side effects of thiamazole and sodium perchlorate treatment were reported during follow-up.

Prevention of ICM-Induced Hyperthyroidism in Euthyroid Goiter
After ICM, the median TSH level in the whole group and in the group without prophylaxis decreased significantly ( $p=0.046$ and $p=0.02$, respectively; Fig. 1 ) and did not change in the group with prophylaxis $(p=0.59$; Fig. 1). Median fT4 values were significantly higher in the group without prophylaxis than in the group with prophylaxis ( $p=0.003$; Table 3 ). There was no statistically significant difference observed in fT3 levels between the groups.

Nineteen patients underwent 1 ICM administration, 10 patients had 2 ICM injections, and 7 patients underwent 3 procedures with ICM. The median time between ICM administration and the first assessment of thyroid hormone levels was 10 days (5.75-19.25). The median follow-up duration was 73 days (40.75-143) in the whole group, and the median time to develop overt hyperthyroidism after the last administered dose of ICM was 17 days (10.5-31.5). The median time of treatment was 52.5 days (24.5-74.25). Values of fT3 and fT4 had normalized in $11 / 17$ (65\%) cases of treated overt hyperthyroidism in the entire group after the median time of 38.5 days (in 2 cases, after 90 days). In patients whose fT 3 and fT 4 levels had normalized, the therapy with thiamazole was discontinued. Six individuals remained hyperthyroid during the entire follow-up despite ATDs treatment, while 1 patient remained hyperthyroid for more than 90 days. Meanwhile, in the case of 5 individuals, follow-up was shorter than 90 days.

The mean size of the thyroid was $38.1( \pm 13.28) \mathrm{mL}$. The thyroid volume of patients who developed hyperthyroidism after ICM was statistically greater than in patients who remained euthyroid after ICM (41.5 vs. 32.9 $\mathrm{mL}, p=0.04$; Fig. 2). 
Table 3. Thyroid status after ICM

\begin{tabular}{lllll}
\hline & $\begin{array}{l}\text { All patients } \\
(n=36)\end{array}$ & $\begin{array}{l}\text { Group with prophylaxis } \\
(n=13)\end{array}$ & $\begin{array}{l}\text { Group without prophylaxis } \\
(n=23)\end{array}$ & $p$ value $^{\mathrm{a}}$ \\
\hline $\mathrm{fT} 4, \mathrm{ng} / \mathrm{dL}$ & $1.69(1.31-2.10)$ & $1.33(1.28-1.54)$ & $1.99(1.52-2.38)$ & 0.003 \\
$\mathrm{fT} 3, \mathrm{pg} / \mathrm{mL}$ & $3.27(2.63-3.61)$ & $2.99(2.36-3.35)$ & $3.27(2.86-4.11)$ & 0.07 \\
$\mathrm{TSH}, \mu \mathrm{IU} / \mathrm{mL}$ & $0.17(0.06-1.40)$ & $1.07(0.42-1.78)$ & $0.08(0.03-0.22)$ & 0.03 \\
\hline
\end{tabular}

Thyroid status was assessed in different points in time during follow-up (between 4 and 505 days after ICM injection). The lowest TSH and the highest thyroid hormone levels measured were used for comparison. Data are shown as medians (interquartile range). Group with prophylaxis, patients who received thiamazole with $(n=6)$ or without $(n=7)$ sodium perchlorate: 1 day prior to ICM and for 14 days after for thiamazole $(20-40$ $\mathrm{mg} /$ daily) and at least 10 days after for sodium perchlorate $(900 \mathrm{mg} /$ daily). Group without prophylaxis, patients who received no prophylactic treatment. ICM, iodinated contrast medium; fT3, free triiodothyronine (reference range: $2.0-4.4 \mathrm{pg} / \mathrm{mL}$ ); fT4, free thyroxine (reference range: $0.93-1.7 \mathrm{ng} / \mathrm{dL}$ ); TSH, thyroid-stimulating hormone (reference range: $0.27-4.2 \mu \mathrm{IU} / \mathrm{mL}$ ). ${ }^{a} p$ value for comparison between group with prophylaxis and group without prophylaxis.

\section{Discussion}

The recommended daily allowance (RDA) for iodine intake for adults is $150 \mu \mathrm{g}$ [1]. The American Thyroid Association advises against the ingestion of iodine in amounts greater than $500 \mu \mathrm{g} /$ day as it may cause thyroid dysfunction such as hypothyroidism, hyperthyroidism, and thyroiditis $[14,15]$. The amount of iodine in ICM considerably exceeds the RDA (e.g., during computed tomography, the patient receives an amount of iodine several thousand times higher than RDA). The elderly and patients with preexisting thyroid disease are among the most susceptible to the adverse effects of iodine excess [3]. In these patients, short-term iodine overload may not cause an impairment (the acute Wolff-Chaikoff effect) but a pathologic increase of thyroid hormone production and consequently IIH [16]. Moreover, the thyroid is exposed to iodine excess long after ICM administration as the urinary iodine concentration may remain elevated for up to 1-2 months after ICM [17]. The prevalence of ICM-induced hyperthyroidism has not yet been well assessed. Marraccini et al. [18] performed a study on patients with ICM exposition during coronary angiography and confirmed ICM-induced hyperthyroidism in $2 \%$ of 1,572 cases. In a retrospective analysis of euthyroid patients performed between 1990 and 2010, Rhee et al. [2] observed that iodine excess after coronary angiography may increase the risk of developing hyperthyroidism up to 2-3 times compared to the control group. A significant limitation of these studies is that hyperthyroidism after ICM administration was diagnosed on the basis of suppressed TSH level only. Hintze et al. [19] found new cases of overt hyperthyroidism in 2 of 788 pa-

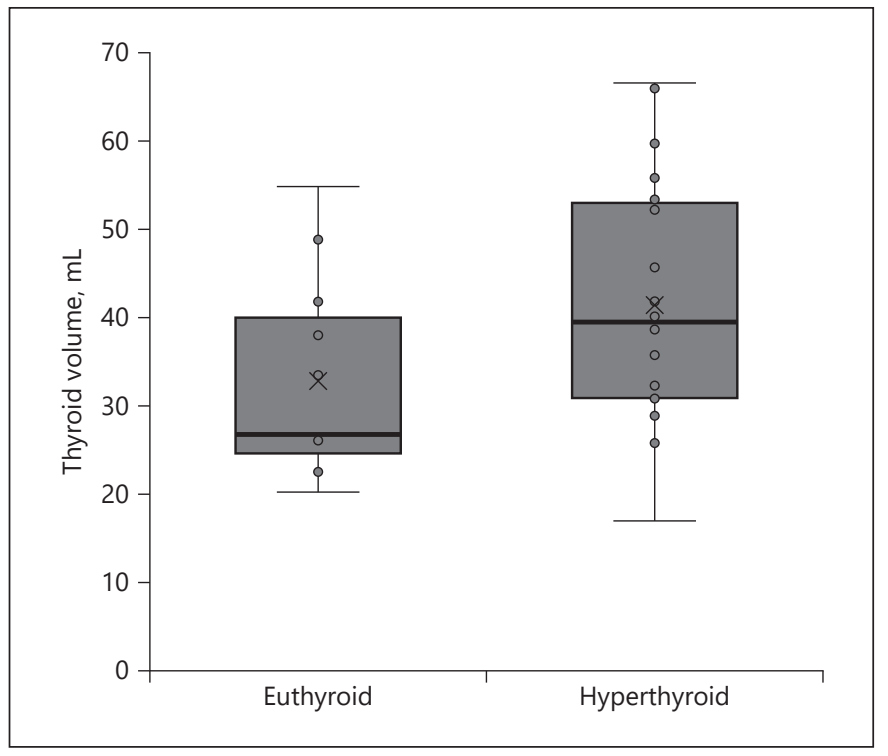

Fig. 2. Thyroid volume according to thyroid status (euthyroid or hyperthyroid) after ICM. Diagram presents thyroid volume compared between patients who developed hyperthyroidism after ICM injection and who remained euthyroid after ICM injection. Data are shown as median values (line across the box) with interquartile (25th-75th percentile) range (the box). Bullets represent individual thyroid volumes. ICM, iodinated contrast medium.

tients (0.25\%) undergoing coronary angiography. A study of patients with ischemic heart disease showed a $10 \%$ risk of hyperthyroidism after ICM in the group of 810 consecutive patients; however, $42 \%$ of cases were also diagnosed with hyperthyroidism at baseline [20]. In a New Zealand [21] and Turkish [22] study, only subclinical hyperthy- 
roidism was observed after ICM with a rate of $2 \%(2 / 102$ cases) and 7\% (7/102) of IIH, respectively. The mentioned studies included unselected patients or those without known thyroid disease, and for that reason, they differ substantially from our study group, which consists only of individuals diagnosed with euthyroid goiter.

Our study confirms that IIH may occur as a complication after radiological procedures using ICM, especially in elderly patients with multinodular goiter. In a tertiary referral center in cardiology, over a period of 5 years from January 2015 to November 2019, we identified 21/36 (58\%) cases of newly developed, clinically significant IIH ( $81 \%$ overt). All patients diagnosed with overt hyperthyroidism were treated with thiamazole. The median time to develop overt hyperthyroidism was 17 days (range 5-73). In most studies, hyperthyroidism was registered after 30 days [20-25]. In those studies, prior thyroid dysfunctions could be overlooked as it was the first assessment of thyroid hormone levels after ICM. In our work, the median duration of ATDs treatment was 52.5 days. Moreover, 6 individuals from the group without prophylaxis remained hyperthyroid during the whole observational period despite ATDs therapy (the longest period being more than 120 days). Our results indicate that ICM-induced hyperthyroidism can persist for months and may require long treatment with ATDs. Similarly, in the study by Bonelli et al. [20], some patients also remained hyperthyroid during follow-up (at 3 and 12 months after ICM) and required treatment with ATDs during this period of time. The levels of fT3 and fT4 had normalized in 11/17 (65\%) cases of treated overt hyperthyroidism in the entire group after the median time of 38.5 days, in 2 cases after 90 days. This confirms that in some patients, IIH may be partially refractory to ATDs treatment, requiring higher doses or prolonged treatment, or the addition of sodium perchlorate.

Patients with thyroid enlargement, especially with multinodular goiter, are at high risk of developing IIH $[16,23,26,27]$. The median age of patients included in our study is 68 years; therefore, the majority was affected by iodine deficiency persisting before 2002 in Poland. We observed that patients who developed hyperthyroidism after ICM had statistically larger thyroid volume than in patients who remained euthyroid after ICM. In another Polish study [23], IIH in patients with multinodular goiter was considered transient, but the size of the thyroid was smaller than our study (15.6 vs. $38.1 \mathrm{~mL}$, respectively). Nolte et al. [24] did not observe any difference in the goiter size between the group that developed hyperthyroidism after ICM and the one that did not.

Prevention of ICM-Induced

Hyperthyroidism in Euthyroid Goiter
Prophylactic therapy of IIH is still a matter of debate. The use of thiamazole and/or sodium perchlorate is recommended by some physicians; however, only a few studies examining the advantages of prophylactic treatment have been performed. There are no specific guidelines concerning the regimen and duration of prophylactic therapy before ICM exposure in clinical practice [28]. The American Thyroid Association does not recommend routine administration of ATDs before ICM for all patients; however, it advises to consider prophylaxis in patients at high risk of developing IIH or with cardiovascular comorbidities [3]. In our work, the occurrence of ICM-induced hyperthyroidism in individuals receiving prophylactic therapy was significantly lower than in the group without prophylaxis (15 vs. 65\%). Moreover, we did not observe any cases of overt hyperthyroidism in the group of patients receiving combined therapy with thiamazole and sodium perchlorate. The individuals who developed overt hyperthyroidism in the group with prophylactic therapy (2/13 patients, $15 \%)$ received only thiamazole as prophylaxis. Fritzsche et al. [29] also showed no cases of hyperthyroidism in the group of euthyroid patients $(n=33)$ on prophylactic treatment with methimazole and perchlorate but administered the prophylaxis in a different regimen: 1 day before and on the day of ICM injection. Nolte et al. [24] studied the influence of prophylactic treatment with 1 group receiving thiamazole and the second receiving sodium perchlorate and concluded that mild hyperthyroidism could not be prevented by monotherapy.

The studied group consists mostly of older patients with many cardiological comorbidities (i.e., congestive heart failure and ischemic heart disease). According to the literature, new onset of hyperthyroidism, even subclinical, is especially life-threatening to the elderly as it is associated with a high risk of incident atrial fibrillation, coronary heart disease, and increased total mortality. The risk of cardiovascular diseases was proven to be increased in untreated hyperthyroid patients and was associated with the duration of decreased TSH [30]. Another recent study showed that early control of hyperthyroidism yields longterm survival benefits [31]. Clinicians should pay special attention to patients with goiter who have cardiological comorbidities or other diseases that require frequent interventions with ICM. In some cases, procedures with ICM may be replaced by alternatives such as magnetic resonance angiography, intravascular imaging techniques for the guidance of percutaneous coronary interventions, and transesophageal or transthoracic echocardiography for the guidance of structural intervention to reduce the need for conventional angiography. All those methods are 
only useful in a stable clinical setting. There is still little alternative to ICM in acute, life-threatening conditions. Afterward, many patients cannot undergo definitive treatment when IIH occurs. After ICM administration, the uptake of RAI by the thyroid gland may be low for many months. In many cases, more than 1 radiological evaluation or hemodynamic intervention with ICM is required, which prolongs low RAI uptake, making the use of RAI treatment impossible [32]. Moreover, some of the cardiovascular conditions may be a contraindication to thyroidectomy. Examining thyroid function before and after ICM administration is not generally recommended [33]. On the basis of our results, we suggest that clinicians should evaluate thyroid status before and 2 weeks after ICM, especially when considering the elderly. We were able to confirm that large nodular goiter is a significant risk factor for developing overt IIH in a country with previous iodine deficiency. Moreover, our study, performed on patients with nodular goiter and multiple cardiac comorbidities, supports the recommendations of the European Society of Urogenital Radiology [28] and the Austrian Thyroid Association [34] to start prophylactic treatment before emergency studies, especially in countries with an iodine deficiency. Before elective studies, a consultation with an endocrinologist is advisable. Combination therapy with thiamazole and perchlorate may be more effective in preventing IIH than the use of thiamazole alone [24].

The present study has some limitations, the main being retrospective study design, small sample groups, treatment of $25 \%$ of patients with low dose thiamazole within 3 months before the admission, and limited follow-up. Prospective, randomized clinical trials with clinically relevant end points (especially concerning cardiovascular mortality) are urgently needed to verify the advantages of the combined prophylactic therapy with both thiamazole and sodium perchlorate and further aid in clinical decision-making regarding patients with ICM-induced hyperthyroidism.
In conclusion, patients with goiter in the euthyroid state are at risk of developing ICM-induced hyperthyroidism. The occurrence of hyperthyroidism after ICM in euthyroid patients with goiter is higher in those with larger thyroid volume. The frequency of ICM-induced hyperthyroidism in euthyroid patients with goiter is lower in those receiving prophylactic therapy with thiamazole in monotherapy or in combination with sodium perchlorate than in those not receiving prophylactic treatment.

\section{Statement of Ethics}

The protocol was approved by the Ethics Committee of the $\mathrm{Na}$ tional Institute of Cardiology in Warsaw, Poland (approval number: IK-NPIA-0021-26/1851/20). Informed consent was waived given the retrospective nature of the study.

\section{Conflict of Interest Statement}

The authors have nothing to disclose. The authors have no conflicts of interest to declare.

\section{Funding Sources}

There are no funding sources to declare.

\section{Author Contributions}

P.M. and K.P. were responsible for the concept and design of the study. P.M., R.W., and K.P. collected patient data. K.P. prepared clinical data for analysis. P.M., T.B., and K.P. analyzed clinical data. K.P. was responsible for statistical analysis, tables, and figures. P.M. and K.P. drafted the manuscript. P.M., R.W., T.B., and K.P. prepared the final manuscript version. All authors edited and approved the final version of the manuscript.

\section{References}

1 World Health Organization, United Nations Children's Fund, International Council for the Control of Iodine Deficiency Disorders. Assessment of iodine deficiency disorders and monitoring their elimination: a guide for programme managers, 3rd ed. Geneva, Switzerland: World Health Organization; 2007. http://whqlibdoc.who.int/publications/2007/ 9789241595827_eng.pdf
2 Rhee CM, Bhan I, Alexander EK, Brunelli SM Association between iodinated contrast media exposure and incident hyperthyroidism and hypothyroidism. Arch Intern Med. 2012; 172(2):153-9.

3 Ross DS, Burch HB, Cooper DS, Greenlee MC, Laurberg P, Maia AL, et al. 2016 american thyroid association guidelines for diagnosis and management of hyperthyroidism and other causes of thyrotoxicosis. Thyroid. 2016; 26(10):1343-421.
4 Zimmermann MB, Boelaert K. Iodine deficiency and thyroid disorders. Lancet Diabetes Endocrinol. 2015;3(4):286-95.

5 Zimmermann MB, Aeberli I, Melse-Boonstra A, Grimci L, Bridson J, Chaouki N, et al. Iodine treatment in children with subclinical hypothyroidism due to chronic iodine deficiency decreases thyrotropin and C-peptide concentrations and improves the lipid profile. Thyroid. 2009;19(10):1099-104 
6 Zimmermann MB, Jooste PL, Pandav CS. Iodine-deficiency disorders. Lancet. 2008; 372(9645):1251-62.

7 Eastman CJ, Zimmermann MB. The Iodine Deficiency Disorders. [Updated 2018 Feb 6]. In: Feingold KR, Anawalt B, Boyce A, et al., editors. Endotext [Internet]. SouthInc Dartmouth (MA): MDText.com; 2000.

8 Szybinski Z; Polish Council for Control of Iodine Deficiency Disorders. Work of the polish council for control of iodine deficiency disorders, and the model of iodine prophylaxis in Poland. Endokrynol Pol. 2012;63(2): 156-60.

9 Trofimiuk-Muldner M, Konopka J, Sokolowski G, Dubiel A, Kiec-Klimczak M, Kluczynski L, et al. Current iodine nutrition status in Poland (2017): is the Polish model of obligatory iodine prophylaxis able to eliminate iodine deficiency in the population? Public Health Nutr. 2020;23(14):2467-77.

10 Zygmunt A, Adamczewski Z, Wojciechowska-Durczynska K, Krawczyk-Rusiecka K, Bieniek E, Stasiak M, et al. Evaluation of the effectiveness of iodine prophylaxis in Poland based on over 20 years of observations of iodine supply in school-aged children in the central region of the country. Arch Med Sci. 2019;15(6):1468-74.

11 Pelewicz K, Miśkiewicz P. Iodinated contrast media-induced hyperthyroidism. JMS. 2020; 89(2):e439.

12 Trzebinska A, Dobruch-Sobczak K, Jakubowski W, Jedrzejowski M. Standards of the polish ultrasound society - update. ultrasound examination of thyroid gland and ultrasound-guided thyroid biopsy. J Ultrason. 2014;14(56):49-60.

13 Gutekunst R, Martin-Teichert H. Requirements for Goiter Surveys and the Determination of Thyroid Size. In: Delange F, Dunn JT, Glinoer D, editors. Iodine Deficiency in Europe NATO ASI Series (Series A: Life Sciences). vol 241: Springer, Boston, MA, USA; 1993.

14 Leung AM, Avram AM, Brenner AV, Duntas LH, Ehrenkranz J, Hennessey JV, et al. Potential risks of excess iodine ingestion and exposure: statement by the american thyroid association public health committee. Thyroid. 2015;25(2):145-6.

15 Institute of Medicine. Dietary reference intakes for vitamin $\mathrm{A}$, vitamin $\mathrm{K}$, arsenic, boron, chromium, copper, iodine, iron, manganese, molybdenum, nickel, silicon, vanadium, and zinc. 2001.

16 Leung AM, Braverman LE. Consequences of excess iodine. Nat Rev Endocrinol. 2014; 10(3):136-42.

17 Lee SY, Chang DL, He X, Pearce EN, Braverman LE, Leung AM. Urinary iodine excretion and serum thyroid function in adults after iodinated contrast administration. Thyroid. 2015;25(5):471-7.

18 Marraccini P, Bianchi M, Bottoni A, Mazzarisi A, Coceani M, Molinaro S, et al. Prevalence of thyroid dysfunction and effect of contrast medium on thyroid metabolism in cardiac patients undergoing coronary angiography. Acta Radiol. 2013;54(1):42-7.

19 Hintze G, Blombach O, Fink H, Burkhardt U, Köbberling J. Risk of iodine-induced thyrotoxicosis after coronary angiography: an investigation in 788 unselected subjects. Eur J Endocrinol. 1999;140(3):264-7.

20 Bonelli N, Rossetto R, Castagno D, Anselmino M, Vignolo F, Parasiliti Caprino M, et al. Hyperthyroidism in patients with ischaemic heart disease after iodine load induced by coronary angiography: Long-term followup and influence of baseline thyroid functional status. Clin Endocrinol. 2018;88(2): 272-8.

21 Jarvis C, Simcox K, Tamatea JA, McAnulty K, Meyer-Rochow GY, Conaglen JV, et al. A low incidence of iodine-induced hyperthyroidism following administration of iodinated contrast in an iodine-deficient region. Clin Endocrinol. 2016;84(4):558-63.

22 Ozkan S, Oysu AS, Kayatas K, Demirtunc R, Eren M, Uslu H, et al. Thyroid functions after contrast agent administration for coronary angiography: a prospective observational study in euthyroid patients. Anadolu Kardiyol Derg. 2013;13(4):363-9.

23 Skorkowska-Telichowska K, Kosinska J, Szymczak R, Tuchendler D, Tuchendler R, Telichowski A, et al. Comparison and assessment of thyroid morphology and function in inhabitants of Lower Silesia before and after administration of a single dose of iodine-containing contrast agent during cardiac intervention procedure. Endokrynol Pol. 2012; 63(4):294-9.
24 Nolte MR, Muller R, Siggelkow H, Emrich D, Hufner M. Prophylactic application of thyrostatic drugs during excessive iodine exposure in euthyroid patients with thyroid autonomy: a randomized study. Eur J Endocrinol. 1996; 134(3):337-41.

25 Conn JJ, Sebastian MJ, Deam D, Tam M, Martin FI. A prospective study of the effect of nonionic contrast media on thyroid function. Thyroid. 1996;6(2):107-10.

26 Roti E, Uberti ED. Iodine excess and hyperthyroidism. Thyroid. 2001;11(5):493-500.

27 Kornelius E, Chiou JY, Yang YS, Lo SC, Peng $\mathrm{CH}$, Lai $\mathrm{YR}$, et al. Iodinated contrast mediainduced thyroid dysfunction in euthyroid nodular goiter patients. Thyroid. 2016;26(8): 1030-8.

28 ESUR Contrast Media Safety Committee. ESUR Guidlines on Contrast Media. Available from: http://www.esur.org/guidelines/ en/index.php\#a.

29 Fritzsche H, Benzer W, Furlan W, Hammerle D, Langsteger W, Weiss P. Prevention of iodine-induced hyperthyroidism after coronary angiography. Acta Med Austriaca. 1993; 20(1-2):13-7.

30 Lillevang-Johansen $M$, Abrahamsen B, Jørgensen HL, Brix TH, Hegedüs L. Duration of hyperthyroidism and lack of sufficient treatment are associated with increased cardiovascular risk. Thyroid. 2019;29(3):332-40.

31 Okosieme OE, Taylor PN, Evans C, Thayer D, Chai A, Khan I, et al. Primary therapy of Graves' disease and cardiovascular morbidity and mortality: a linked-record cohort study. Lancet Diabetes Endocrinol. 2019;7(4):278-87.

32 Silberstein EB, Alavi A, Balon HR, Clarke SE, Divgi C, Gelfand MJ, et al. The SNMMI practice guideline for therapy of thyroid disease with 131I 3.0. J Nucl Med. 2012;53(10):163351.

33 Windecker S, Kolh P, Alfonso F, Collet J-P, Cremer J, Falk V, et al. 2014 ESC/EACTS Guidelines on myocardial revascularization. EuroIntervention. 2015 Jan; 10(9):1024-94.

34 Österreichische Schilddrüsengesellschaft. Röntgenkontrastmittel bei Patienten mit Risiko jodinduzierter Schilddrüsenfunktionsstörungen. May 2016. https://www. schilddruesengesellschaft.at/rontgenkontrastmittel-und-schilddruse- 0 .
Prevention of ICM-Induced

Hyperthyroidism in Euthyroid Goiter
Eur Thyroid J 2021;10:306-313

DOI: $10.1159 / 000515126$ 